\title{
Recognition of Bread Key Odorants by Using Polymer Coated QCMs
}

\author{
Takashi Nakai Student Member (The Uneversity of Tokyo) \\ Shinji Kouno Non-member (Mayekawa MFG. Co., Ltd) \\ Naoya Hiruma Non-member (Mayekawa MFG. Co., Ltd) \\ Masaki Shuzo Member (The Uneversity of Tokyo) \\ Jean-Jacques Delaunay Non-member (The Uneversity of Tokyo) \\ Ichiro Yamada Non-member (The Uneversity of Tokyo)
}

Keywords : QCM, polymer film, key odorant, food quality, bread, LSER

\section{Introduction}

It is now known that deterioration of bread quality with storage time is accompanied by a drastic decrease in the concentration of 2-acetyl-1-pyrroline ( $90 \%$ in 4 days), a key pleasant roasty odor, and little change in the concentration of (E)-2-nonenal, an infamous unpleasant fatty odor ( $14 \%$ in 4 days). In this way, deterioration of bread quality during storage may be correlated with the rapid loss of key odorants of fresh bread.

Major challenges in applying odor sensing to the recognition of key odorants and the evaluation of bread quality are the realization of high-sensitivity and high-selectivity sensors. The main objective of this study was to develop and evaluate odor sensors for recognition of roasty odors and fatty odors found in bread.

\section{Experimental Procedure}

In order to select an optimum coating material, we used the Linear Solvation Energy Relationship (LSER) equation as a tool to quantify coating material solubility properties. Polyisobutylene (PIB) polymer and methylphenylsiloxane (25\%) diphenylsiloxane (75\%) copolymer (OV25) which were chosen by using LSER method were coated on Quartz Crystal Microbalance (QCM) sensors and used in recognition of bread key odorants.
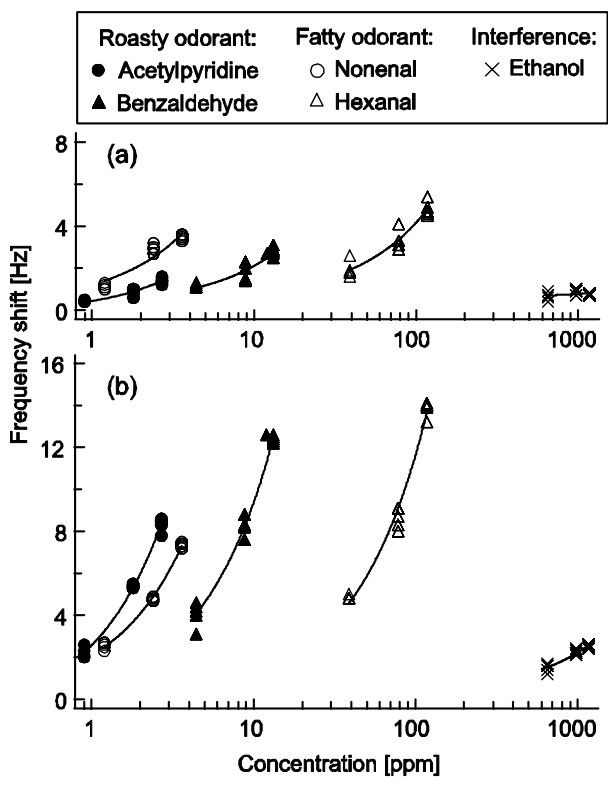

Fig. 1. Sensor responses of the (a) PIB- and (b) OV25-coated QCMs to key odorants and interference compounds
Representative compounds of key roasty odorants of bread were taken as 3-acetylpyridine and benzaldehyde, and representative key fatty odorants were hexanal and (E)-2-nonenal.

\section{Results and Discussion}

Both OV25- and PIB-coated QCM fabricated sensors could detect concentration as low as $0.9 \mathrm{ppm}$ of 3-acetylpyridine and 1.2 ppm of (E)-2-nonenal. The sensitivity to 3-acetylpyridine of the OV25-coated QCM was about 1000 times higher than that of ethanol, the major interference compound in bread key odorant analysis (Fig. 1). Further, the OV25-coated QCM response was 5-6 times and 2-3 times larger than that of the PIB-coated QCM when exposed to roasty odorants and to fatty odorants, respectively. The odor recognition map representing the frequency shifts of the OV25-coated QCM against the frequency shift of the PIB-coated QCM (Fig. 2). The difference in sensitivity of the OV25- and PIB-coated QCMs which we fabricated made possible to discriminate roasty from fatty odorants.

\section{Conclusion}

Based on LSER calculations, we designed and realized polymer coated QCM sensors. We found that the combination of an OV25-coated QCM and a PIB-coated QCM was successful in discriminating roasty odorants from fatty odorants at the ppm level and the undesirable effect of ethanol one of the major interference vapors in the analysis of bread quality could be alleviated. These encouraging results suggest the possibility of applying our approach to the evaluation of bread quality.

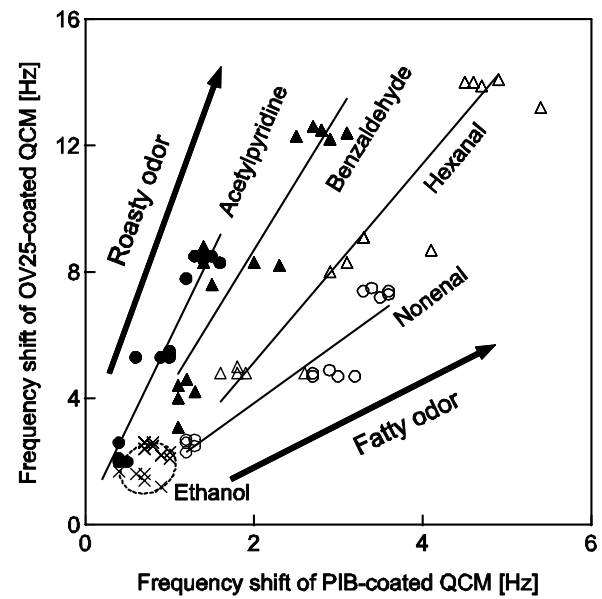

Fig. 2. Classification of key odorants and interference compounds by using PIB- and OV25-coated QCMs 


\section{Recognition of Bread Key Odorants by Using Polymer Coated QCMs}

$\begin{array}{ll}\text { Takashi Nakai* } & \text { Student Member } \\ \text { Shinji Kouno** } & \text { Non-member } \\ \text { Naoya Hiruma** } & \text { Non-member } \\ \text { Masaki Shuzo* } & \text { Member } \\ \text { Jean-Jacques Delaunay* } & \text { Non-member } \\ \text { Ichiro Yamada* } & \text { Non-member }\end{array}$

Polyisobutylene (PIB) polymer and methylphenylsiloxane (25\%) diphenylsiloxane (75\%) copolymer (OV25) were coated on Quartz Crystal Microbalance (QCM) sensors and used in recognition of bread key odorants. Representative compounds of key roasty odorants of bread were taken as 3-acetylpyridine and benzaldehyde, and representative key fatty odorants were hexanal and (E)-2-nonenal. Both OV25- and PIB-coated QCM fabricated sensors could detect concentration as low as 0.9 ppm of 3-acetylpyridine and $1.2 \mathrm{ppm}$ of (E)-2-nonenal. The sensitivity to 3-acetylpyridine of the OV25-coated QCM was about 1000 times higher than that of ethanol, the major interference compound in bread key odorant analysis. Further, the OV25-coated QCM response was 5-6 times and 2-3 times larger than that of the PIB-coated QCM when exposed to roasty odorants and to fatty odorants, respectively. The difference in sensitivity of the OV25- and PIB-coated QCMs we fabricated made possible to discriminate roasty from fatty odorants as was evidenced by the odor recognition map representing the frequency shifts of the OV25-coated QCM against the frequency shift of the PIB-coated QCM. In conclusion, we found that the combination of an OV25-coated QCM and a PIB-coated QCM was successful in discriminating roasty odorants from fatty odorants at the ppm level.

Keywords : QCM, polymer film, key odorant, food quality, bread, LSER

\section{Introduction}

Quality of bread is commonly evaluated by sensory test usually involving trained flavor panelists. This evaluation method highly relies on skilled human and therefore has important drawbacks such as high costs, bias and large statistical errors. Also the sensory test method cannot be applied in real time and therefore is not adequate for the on-the-site optimization of bread recipes and processing parameters that is so needed to improve product quality in food processing industry. Thus, a sensing system approach to bread qualty evaluation is highly needed as an alternative to sensory tests.

Bread odor is one of the possible quality indicators that could be automatically monitored by newly developed odor sensor systems. It is now known that deterioration of bread quality with storage time is accompanied by a drastic decrease in the concentration of 2-acetyl-1-pyrroline ( $90 \%$ in 4 days), a key pleasant roasty odor, and little change in the concentration of (E)-2-nonenal, an infamous unpleasant fatty odor (14\% in 4 days). Futhermore, it was found from sensory tests that the bread crust loses its pleasant roasty odor and that a fatty stale odor note develops during storage ${ }^{(1)}$. In this way, deterioration of bread quality during storage may be correlated with the rapid loss of key odorants of fresh bread (e.g. 2-acetyl-1-pyrroline) and the retention of odorants associated with stale bread (e.g.

* Department of Engineering Synthesis, School of Engineering, The University of Tokyo, 7-3-1 Hongo, Bunkyo-ku, Tokyo 113-8656

** Mayekawa MFG. Co., Ltd., 2-13-1 Botan, Koto-ku, Tokyo $135-8482$
(E)-2-nonenal) ${ }^{(1)(2)}$. In this study, we aimed at developing odor sensors to recognize the key odorants causing the roasty and fatty notes in the flavor of breads. 2-Acetyltetrahydropyridine and acetylpyrazine were reported as major roasty odorant compounds whereas (E)-2-octenal and (E,E)-2,4-decadienal were reported as major fatty odorant compounds ${ }^{(3)(4)}$. These major key odorants were chosen as the target compounds for our odor sensor system.

Major challenges in applying odor sensing to the recognition of key odorants and the evaluation of bread quality are the realization of high-sensitivity and high-selectivity sensors. Bread odor comprises more than 540 Volatile Organic Compounds (VOCs) ${ }^{(5)}$. Moreover, key odorants are known to have very low concentration on the order of a few micrograms per kilogram of bread. The major interference compounds, ethanol and acetic acid, have concentrations larger than the order of milligrams per kilogram ${ }^{(5)}$. Water vapor also belongs to the interference compounds as it is found in bread and in the environment as well. All other compounds including malty odorant compounds such as methylpropanal and buttery odorant compounds such as 2,3-butanedione will be regarded as interference compounds in this study because we aim at assessing bread quality from change in the balance of roasty and fatty odorants ${ }^{(4)}$.

We used Quartz Crystal Microbalance (QCM) and coated one of the QCM electrodes with a rubbery polymer film which acts as selective sorption sites for odor molecules. Polymer coated QCMs offer great flexibility as odor sensors because polymers can be given intended molecular recognition functions. Moreover, polymer coated-QCMs are known to exhibit fast response times ${ }^{(6)}$.

The main objective of this study was to develop and evaluate 


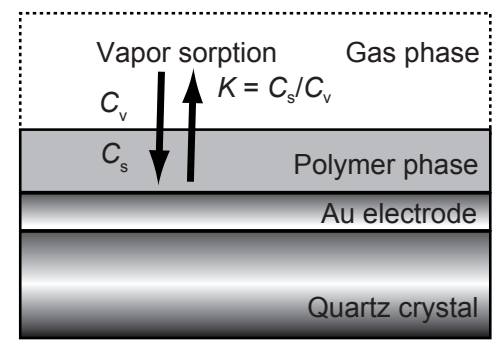

Fig. 1. Schematic of vapor sorption at the interface air/polymer

Table 1. $\log K$ values $(298 \mathrm{~K})$ of several commercially available polymers for the studied key odorants and interference compounds ${ }^{(8)(9)}$

\begin{tabular}{lcccccc}
\hline & \multicolumn{2}{c}{ Roasty odorant } & \multicolumn{2}{c}{ Fatty odorant } & \multicolumn{2}{c}{ Interference } \\
\cline { 2 - 7 } Polymer & $\begin{array}{c}\text { Acetyl } \\
\text { pyridine }\end{array}$ & $\begin{array}{c}\text { Benz } \\
\text { aldehyde }\end{array}$ & $\begin{array}{c}\text { (E)-2- } \\
\text { Octenal }\end{array}$ & Hexanal & Ethanol & Water \\
\hline PIB & 4.55 & 3.60 & 4.42 & 2.86 & 0.93 & -0.20 \\
OV25 & 5.52 & 4.30 & 4.75 & 3.19 & 1.47 & 0.57 \\
PECH & 5.92 & 4.56 & 4.95 & 3.43 & 2.07 & 1.64 \\
SIL & 6.91 & 5.58 & 5.81 & 4.31 & 3.48 & 3.90 \\
\hline
\end{tabular}

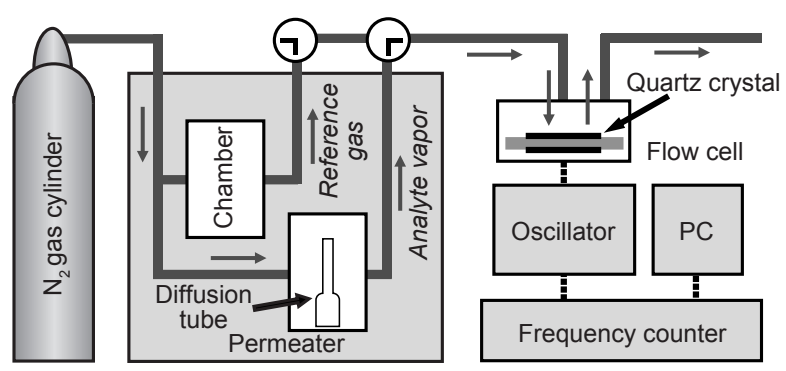

Fig. 2. Schematic of the odor sensing experimental setup

polymer coated QCM odor sensors for recognition of roasty odors and fatty odors found in bread.

\section{Experimental Procedure}

2.1 Methodology We used $5 \mathrm{MHz}$ AT-cut quartz crystals with $\mathrm{Au}$ electrodes. Coating material on the electrodes greatly influences the sensitivity and selectivity of QCM sensors. The partition coefficient, $K$, is established as a means of quantifying the total sorption to which the interaction between vapor and absorbent material contributes. The partition coefficient represents the ratio between the concentration of the vapor in the absrobent material (such as polymer), $C_{\mathrm{s}}$, and the concentration of the vapor in the gas phase, $C_{\mathrm{v}}$, as described in the schematic of Fig. 1. In other words, the $K$ values indicate the ability of molecular recognition of a polymer. The ideal coating material, in our aimed odor sensor, should have high $K$ values for key odorants and low $K$ values for interference compounds.

In order to estimate $K$ values, we used the Linear Solvation Energy Relationship (LSER) method as a tool to quantify the various interactions between vapors and polymers ${ }^{(7)}$. The LSER method are based on a set of solvation parameters describing the solubility properties of vapors. LSER equation gives an estimate of the $\log K$ values as a linear combination of terms related to fundamental interactions and solubility properties characterized by
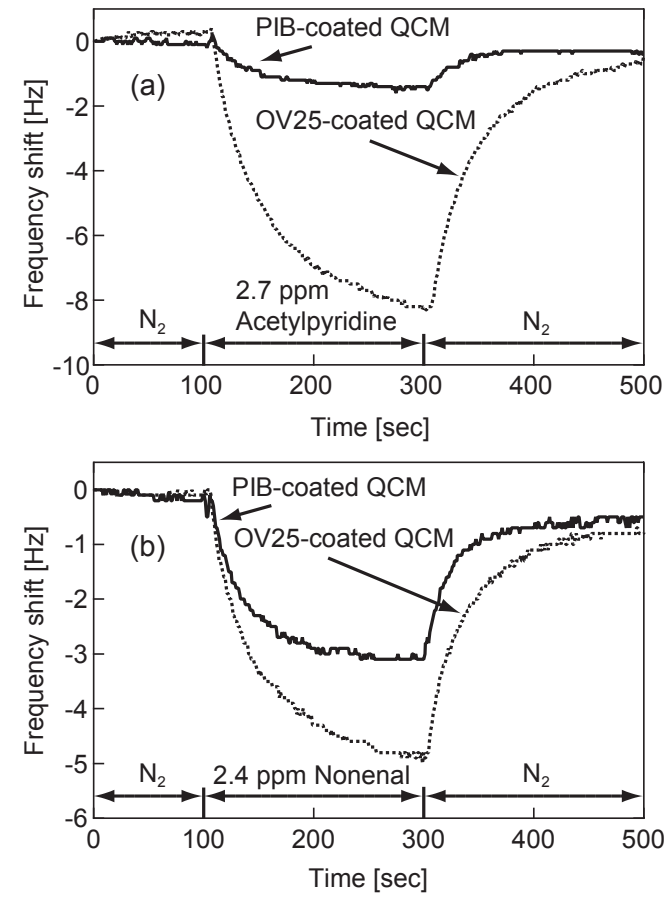

Fig. 3. Response curve of PIB- and OV25-coated QCM to (a) $2.7 \mathrm{ppm} 3$-acetylpyridine and (b) $2.4 \mathrm{ppm}$ (E)-2-nonenal

solvation parameters of vapors and coefficients of polymers ${ }^{(6)(7)}$. The solvation parameters we used were obtained at $298 \mathrm{~K}$ from theoritical estimates ${ }^{(8)}$ and the coefficients of the employed polymers were obtained at $298 \mathrm{~K}$ from experimental work using a gas chromatographic method ${ }^{(9)}$.

2.2 Proposal of Odor Sensor for Bread The 6 compounds listed in Table 1 were used to select an optimum coating material. Unfortunately, the solvation parameters of 2-acetyl-1-pyrroline and (E)-2-nonenal have not been fully reported. We used compounds with same chemical functions and similar odors for which the solvation parameters are known. 3 -acetylpyridine was used as "pleasant roasty" key odorant in place of 2-acetyl-1-pyrroline because many heterocyclic compouds with the nitrogen atom and side-chaine acetyl group such as 2-actyl-1-pyrroline, 2-acethylpyrazine exhibit biscuit- or cracker-like odors $^{(10)}$. (E)-2-octenal which has fatty odor and is similar in structure to (E)-2-nonenal was used as an alternative to (E)-2-nonenal. Since benzaldehyde smells like almond or burnt sugar and hexanal smells green or fatty similar to (E)-2-nonenal, benzaldehyde and hexanal were used as roasty and fatty odorants, respectively. Ethanol and water were used as the major interference compounds.

The computed $\log K$ values at $298 \mathrm{~K}$ of several commercially available polymers for the studied key odorants and interference compounds obtained by the LSER equation ${ }^{(8)(9)}$ are listed in Table 1. Under the condition of low sensitivity to the interference compounds, neither polyepichlorohydrin (PECH) nor poly (biscyanopropyl siloxane) (SIL) is suitable to our objective sensor, although these two polymers have excellent sensitivity to 3-acetylpyridine and (E)-2-octenal. Polyisobutylene (PIB) and methylphenylsiloxane (25\%) diphenylsiloxane $(75 \%)$ copolymer (OV25) have good selectivity toward key odorants and at the same time low sensitivity to the major interference compounds (ethanol 


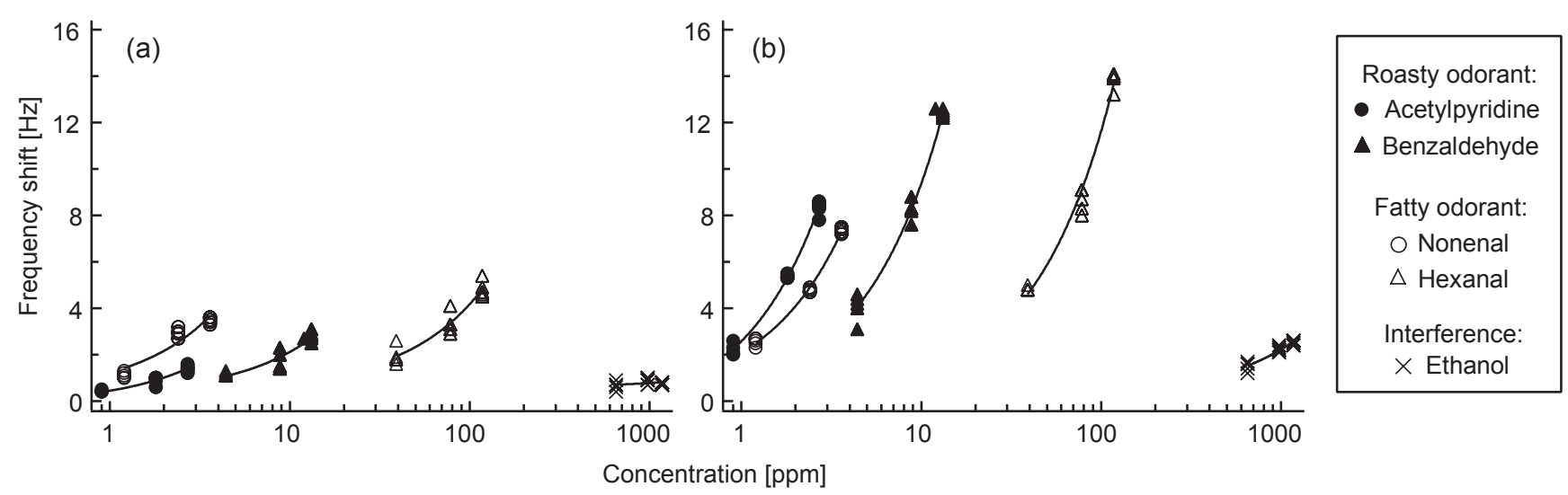

Fig. 4. Sensor responses of the (a) PIB- and (b) OV25-coated QCMs to key odorants and interference compounds

and water). Further, the $\log K$ values of PIB and OV25 for the malty interference compound methylpropanal are 1.59 and 2.05 , respectively. These $\log K$ values are much lower than those for key odorants suggesting low sensitivity to interference compounds. For the key roasty and fatty odorants however much higher sensitivities are expected. The $K$ value for 3-acetylpyridine of OV25 is nearly 10 times larger than that of PIB, and the $K$ value for (E)-2-octenal of OV25 is only about 2 times larger than that of PIB. This difference in the two polymer sensitivities suggests the possibility of recognition of roasty and fatty odor by using OV25and PIB-coated QCMs in combination.

2.3 Odor Sensing Experiment To evaluate our fabricated sensors, we measured the response of the polymer coated QCM upon exposure to 3-acethylpyridine, benzaldehyde (roasty), (E)-2-nonenal, hexanal (fatty) and ethanol (interference). The variation in the QCM resonance frequency upon exposure to these compounds for 200 seconds was recorded with a resolution of $0.1 \mathrm{~Hz}$ using the setup of Fig. 2. Pure $\mathrm{N}_{2}$ was used as carrier gas for the analyte as well as reference gas. The analyte vapor concentration in the carrier gas was accurately controlled by a gas generation apparatus (Double chamber permeater PD-1B-2, Gastec). The analyte vapor concentration relative to the carrier gas can be controlled by varying the flow and the temperature of the carrier gas as well as the size of the diffusion tube which contained the analyte. In this study, the concentration of the vapors was adjusted by changing the number and type of the diffusion tubes, the flow and temperature of the carrier gas being kept constant. The gas flow was set to $0.20 \mathrm{~L} / \mathrm{min}$ and the temperature of the diffusion tube was $30^{\circ} \mathrm{C}$. The second chamber of our permeater was used to generate a flow of carrier gas at the same temperature as that of the flow of analyte vapor in carrier gas, so that upon switching from a carrier gas flow without and with analyte vapor no temperature difference betwwen the two flows was generated. The volume of the QCM flow cell was $150 \mu \mathrm{L}$. All experiments were performed at room temperature $\left(23-24^{\circ} \mathrm{C}\right)$. The sensor responses of the OV25- and PIB-coated QCMs were measured for 3-acetylpyridine concentration in the range of 0.9 to $2.7 \mathrm{ppm}$, for benzaldehyde in the range of 4.4 to $13.2 \mathrm{ppm}$, for (E)-2-nonenal in the range of 1.2 to $3.6 \mathrm{ppm}$, for hexanal in the range of 39 to $117 \mathrm{ppm}$ and for ethanol in the range of 650 to 1170 ppm.

\section{Results and Discussion}

3.1 Polymer Coating The OV25 and PIB polymers

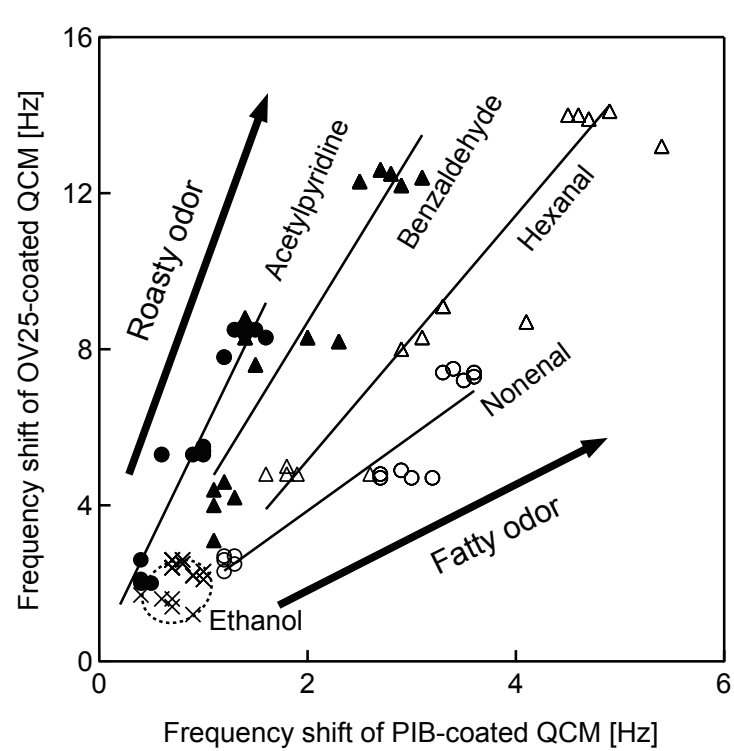

Fig. 5. Classification of key odorants and interference compounds by using PIB- and OV25-coated QCMs

were prepared in cyclohexane solution with $0.25 \% \mathrm{w} / \mathrm{v}$ polymer concentration and sprayed on QCMs (Resist spray coater RS-C610L, TechInTech Corp.). When a rubbery polymer coated QCM is used as a pure mass sensor for vapors, the maximum thickness of the polymer coating on the electrode surface is limited by the viscoelastic effects of the coating. In general, the film thickness should be limited to within $0.1-0.3 \%$ of the acoustic wavelength $^{(11)}$, which in the case of a $5 \mathrm{MHz}$ resonance frequency is $660 \mu \mathrm{m}$. The polymer layer thickness was chosen to be about $2 \mu \mathrm{m}$.

According to the Sauerbrey equation ${ }^{(12)}$, the film thickness is characterized by the frequency shift generated by the polymer coating. The frequency shift observed by the OV25 coating and the PIB coating were $-12 \mathrm{kHz}$ and $-11 \mathrm{kHz}$, respectively. Under the assumption of homogeneity of the fabricated layers, the thickness of the OV25 and PIB layers can be estimated to be just smaller than $2 \mu \mathrm{m}$.

3.2 Sensor Response Our developed sensors could detect 0.9 ppm 3-acetylpyridine and $1.2 \mathrm{ppm}$ (E)-2-nonenal. Examples of the recorded sensor responses for $2.7 \mathrm{ppm}$ 3-acetylpyridine and $2.4 \mathrm{ppm}$ (E)-2-nonenal are reproduced in Fig. 3. The observed sensor responses showed a good stability 
(baseline drift of less than $0.1 \mathrm{~Hz} / \mathrm{s}$ ) and a good reversibility (resonance frequency recovers to almost its original value). The detection limit of the shift in the resonance frequency was estimated to be $0.4 \mathrm{~Hz}$.

The measured frequency shifts caused by sorption of key odorant and interference vapors were plotted in Fig. 4. The resulting frequency shift showed a good reproducibity. For the roasty odorants, we found that the frequency shifts of the OV25-coated QCM was about 5-6 times larger than that of the PIB-coated QCM. The log $K$ value obtained from our experiment for the benzaldehyde agreed well with the theoritical value, whereas the agreement was poor for the acetylpyridine. For fatty odorants, the frequency shift of the OV25-coated QCM was about 2-3 larger than that of the PIB-coated QCM. Good agreement between the $\log K$ values obtained from LSER theory and experiment for hexanal was obtained. It was thus found that OV25 exhibited much stronger interactions for roasty odorants than PIB and almost as strong interactions as PIB for fatty odorants. This is well explained by the structure of the roasty odorants with heterocyclic or aromatic group and that of the fatty odorants with straight-chain aldehyde compounds. Finally, it can be said that Fig. 4 revealed good selectivity to key odorants for OV25- and PIB-coated QCMs The frequency shifts of the OV25- and PIB-coated QCMs upon exposure to $975 \mathrm{ppm}$ ethanol were of similar magnitude as that for exposure to $0.9 \mathrm{ppm} 3$-acetylpyridine. Consequently, the polymer coated QCMs which we developed featured sensitivity to 3-acetylpyridine about 1000 times larger than that for ethanol. Note that the theoritical sensitivity ratios between 3-acetylpyridine and ethanol obtained from the LSER equation were even larger. This disagreement between computed and observed ratios was very likely explained by the difficulty to correctly estimate the very small sensitivities to ethanol of our sensors.

3.3 Recognition of Key Odorants Our study aimed at the recognition of key odorants. An odor recognition map was constructed by plotting the frequency shifts of the OV25-coated QCM against the frequency shifts of the PIB-coated QCM (Fig. 5). In this map, the roasty odorants such as 3-acetylpyridine and benzaldehyde grouped in the upper left side of the map, whereas the fatty odorants such as (E)-2-nonenal and hexanal grouped in the lower right side of the map. Roasty odorant group and fatty odorant group were clearly separated at high concentration. However, the ethanol interference compound was found to lie nearby roasty odorants at low concentration. As long as the concentration of ethanol remains lower than $1000 \mathrm{ppm}$, the roasty odorants could be discriminated.

Consequently, it could be seen that the odor quality of bread can be determined by using our developed QCM odor sensors at the ppm concentration level of key odorants. It is suggested that our developed QCM odor sensors could recognize different odorants on the basis of differences in odorant molecular structures that were well accounted for by the LSER model.

However, the sensors developed in this study are not sensitive enough to detect the studied key odorants at the concentration level found in bread head space which can be as low as ppb (e.g. the nonenal rate of evaporation in the headspace of baguettes was found as low as $\left.0.5 \mathrm{ng} / \mathrm{L} / \mathrm{min}^{(13)}\right)$. Therefore, future work is needed to enhance the sensitivity of the sensors, for example by using quartz crystals with higher resonance frequencies and/or a pre-concentration technique ${ }^{(14)}$.

\section{Conclusion}

Based on LSER calculations, we designed and realized polymer coated QCM sensors that can be used in the recognition of the key odorants of bread with limited effects of the interference compounds. OV25 and PIB polymers were selected as the selective sorption materials and layers of these polymers were deposited on QCM electrodes by using spray coating. The developed OV25- and PIB-coated QCMs could detect concentration as low as $0.9 \mathrm{ppm} 3$-acetylpyridine and $1.2 \mathrm{ppm}$ (E)-2-nonenal. The sensitivity to the key odorant 3-acetylpyridine was about 1000 times that of ethanol a major interference vapor. The response of the OV25-coated QCM was 5-6 times larger than that of the PIB-coated QCM for exposure to roasty odorants. The two developed sensors when used in combination could discriminate roasty from fatty odors at the ppm concentration level. The undesirable effect of ethanol one of the major interference vapors in the analysis of bread quality could be alleviated by careful selection of the polymers. These encouraging results suggest the possibility of applying our approach to the evaluation of bread quality.

\section{Acknowledgements}

We wish to thank Toshio Ide of TechInTech Corporation for fruitful discussions and valuable support in the deposition of polymer layers by spray coating.

(Manuscript received Aug. 31, 2007, revised Nov. 19, 2007)

\section{References}

(1) P. Schieberle and W. Grosch : "Changes in the concentration of potent odourants during storage of white bread", Flavour and Fragrance Journal, Vol.7, pp.213-218 (1992)

(2) P. Schieberle and W. Grosch : "Potent odorants of the wheat bread crumb Differences to the crust and effect of a longer dough fermentation", European Food Research and Technology, Vol.192, No.2, pp.130-135 (1991)

(3) M. Rychlik and W. Grosh : "Identification and quantification of potent odorants formed by toasting of wheat bread", Lebensmittel-Wissenschaft und-Technologie, Vol.29, No.5-6, pp.515-525 (1996)

(4) G. Zehentbauer and W. Grosch : "Crust aroma of baguettes, I. Key odorants of baguettes prepared in two different ways", Journal of Cereal Science, Vol.28, No.1, pp.81-92 (1998)

(5) J. A. Ruiz, J. Quilez, M. Mestres, and J. Guasch : "Solid-phase microextraction method for headspace analysis of volatile compounds in bread crumb", Cereal Chemistry, Vol.80, No.3, pp.255-259 (2003)

(6) J. W. Grate : "Acoustic wave microsensor arrays for vapor sensing", Chemical Reviews, Vol.100, No.7, pp.2627-2647 (2000)

(7) J. W. Grate and M. H. Abraham : "Solubility interactions and the desigh of chemically selective sorbent coatings for chemical sensors and arrays", Sensors and Actuators B: Chemical, Vol.3, No.2, pp.85-111 (1991)

(8) M. H. Abraham, J. Adonian-Haftvan, G. S. Whitig, A. Leo, and R. S. Taft : "Hydrogen bonding part 34. The factors that influence the solubility of gases and vapors in water at $298 \mathrm{~K}$ and a new method for its determination”, Journal of Chemical Society Perkin Transaction 2, pp.1777-1791 (1994)

(9) J. W. Grate, S. J. Patrash, and M. H. Abraham : "Method for estimating polymer-coated acoustic-wave vapor sensor responses", Analytical Chemistry, Vol.67, No.13, pp.2162-2169 (1995)

(10) T. H. Parliament, R. J. Mcgorrin, and C. T. Ho : "Thermal Generation of Aromas", American Chemical Society, Washington, DC (1989)

(11) K. Bodenhofer, A. Hierlemann, G. Noetzel, U. Weimar, and W. Gopel : "Performances of mass-sensitive devices for gas sensing: Thickness shear mode and surface acoustic wave transducers", Analytical Chemistry, Vol.68, No.13, pp.2210-2218 (1996)

(12) G. Sauerbrey : "Verwendung von Schwingquarzen zur Wagung dunner Schichten und zur Mikrowagung", Zeitschrift für Physik, Vol.155, No.2, pp.206-222 (1959)

(13) G. Zehentbauer and W. Grosch : "Apparatus for quantitative headspace analysis of the characteristic odorants of baguettes", Zeitschrift fur Lebensmittel-Untersuchung und-Forschung A: Food Research and 
Technology, Vol.205, No.4, pp.262-267 (1997)

(14) T. Nakamoto, K. Kobayashi, and J. Ito : "Study of halitosis-substance sensing using electrochemical sensor array combined with a preconcentrator", Proceedings of the 21st Sensor Symposium, pp.143-146 (2004)

Takashi Nakai

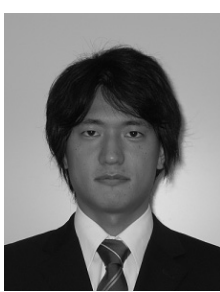

(Student Member) was born in Tokyo, Japan, on August 13, 1982. He received the B.E. degree in engineering synthesis from The University of Tokyo in 2005 . He is currently a master course student in School of Engineering, the University of Tokyo. He has worked on research and development of gas sensors.

Shinji Kouno

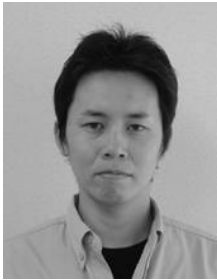

(Non-member) was born in Hiroshima, Japan, on December 18, 1976. He received the master degree in biosphere science from Hiroshima University in 2001, and is presently an assistant manager at Mayekawa MFG. Co., Ltd. He has worked on chemical analysis of food.

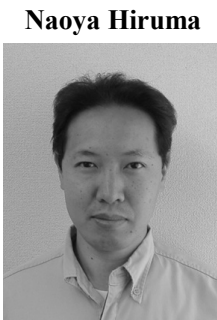

(Non-member) was born in Saitama, Japan, on June 16, 1969. He received the master degree in Department of Agriculture of the Meiji University Graduate School in 1994, and is presently an assistant general manager at Mayekawa MFG. Co., Ltd. He has worked on quality and evaluation of food.
Masaki Shuzo

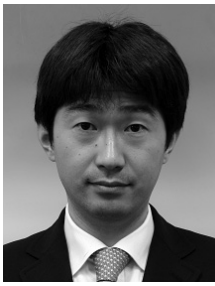

(Member) was born in Nara, Japan, on July 23, 1974. He received the Ph.D. degree in mechano-informatics from the University of Tokyo in 2003. He received a JSPS research fellowship for young scientists in 2000-2003 and 2004-2007. He is presently an assistant professor of the University of Tokyo. He has worked on research and development of biological MEMS. The Robotics Society of Japan member.

Jean-Jacques Delaunay (Non-member) was born on April 18, 1967. He

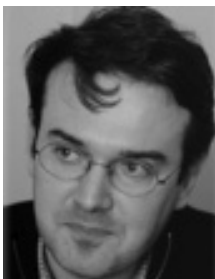
received the Engineer degree from Strasbourg National School of Engineering and his Ph.D. degree from the University Louis Pasteur. He is currently an associate professor at the University of Tokyo. His research interests include sensors/devices based on nanometer-scale structured materials and their applications in environmental monitoring and forecasting systems.

Ichiro Yamada

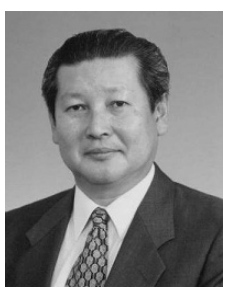

(Non-member) was born in Nagoya, Japan, on August 1, 1949. He received the Ph.D. degree in mechanical engineering from The University of Tokyo in 1985, and is presently a professor of the University of Tokyo. He has worked on research and development of information and energy systems, sensors and sensor networks, and new communication systems. Japan Society of Mechanical Engineers, Institute of Electronics, Information and Communication Engineers, Japan Society for Precision Engineers, etc. 